Proceedings

\title{
Unifying the Concepts of Information: Methodological Solution ${ }^{\dagger}$
}

\author{
Yixin Zhong \\ College of Artificial Intelligence, Beijing University of Posts and Telecommunications, Beijing 100876, China; \\ zyx@bupt.edu.cn; Tel.: +86-1370-123-0645 \\ † Conference Theoretical Information Studies (TIS), Berkeley, CA, USA, 2-6 June 2019. \\ Published: 8 May 2020
}

\begin{abstract}
What are the appropriate concepts of information? This is an old issue, yet which has to be the No.1 and cannot be ignored in the study of information science. Diverse controversies and confusions in the studies of information are resulted from different understandings of the concept of information. This fact clearly indicates that the real root, which leads to the diversity of approaches, is due to the "divide and conquer" methodology. We suggest here a novel understanding of the concept of information. At first, a group of popular definitions of information are analyzed. Then, the system methodology is applied to the diversity of the definitions with the goal of unification. Based on this methodology, an ecological model for information is established and the systematic definitions of information are hence derived from this model.
\end{abstract}

Keywords: methodological challenge; ecological model of information process; ontological information; and epistemological information

\section{Introduction}

A precise understanding of the concept of information is by all means the most fundamental issue in the studies of information. However, researchers with different backgrounds and different viewpoints have already formulated a diversity of definitions on information in different fields, such as Shannon's understanding of information in communication, Wiener's definition in cybernetics and cognitive science, Ashby's understanding in biological control theory, and Bateson's definition in psychology, and so on. This situation reveals that the "divide and conquer" methodology is the cause of the existence of different definitions of information.

Is it possible to have the precise definition and the common understanding of the concept of information for all different fields of information discipline? What would then be the effective means for the unity in the diversity of the existing concepts of information?

To solve these problems, we need a novel attitude. The right approach is to eliminate the unnecessary diversity, which comes from the "divide and conquer" methodology, and to apply the new one instead.

The rest of the paper is organized as follows. In Section 2, reviews and comments on some typical definitions of information are made and the improper methodological issue governing the information defining is revealed. In Section 3, the methodology that is suitable for the information studies is summarized and the ecological model of information process is thus established. In Section 4, the new definitions of information that are able to unify the other definitions are derived from the new methodology and new model. Finally, some concluding remarks concerning the model and the definitions are made in Section 5. 


\section{Reviews and Comments on Typical Existing Definitions}

Researchers introduced numeral definitions of information. Here, we list just a few of them. It will be helpful to review what has been achieved in history and what is still insufficient, or even unsuitable, for properly understanding the concept of information.

In his monograph "Cybernetics, or Control and Communication in the Animal and the Machine" published in 1948, the founder of cybernetics Norbert Wiener made a famous statement that information is itself neither matter nor energy [1]. To the author's knowledge, it is this statement that distinguished clearly the concept of information from those of matter and energy for the first time in the history of science. However, he did not say anything about what information is in this statement. This opened up a very wide room for various attempts of defining information afterwards.

In his monograph "Cybernetics and Society" published in 1950, Wiener tried to give a definition of information, saying that information is the content of what was exchanged during the interaction between humans and the environment [2]. But this is not completely true, because what humans exchange with their environment is not merely information, but also matter and energy. This definition of information clearly is not precise.

In his seminal paper "A Mathematical Theory of Communication", published in 1948, the founder of information theory Claude Shannon proposed the equation for the mutual information calculation [3]:

$$
\mathrm{I}(\mathrm{X}, \mathrm{Y})=\mathrm{H}(\mathrm{X})-\mathrm{H}(\mathrm{X} \mid \mathrm{Y})
$$

where $H(X)$ is the amount of uncertainty the recipient has before communication and $H(X \mid Y)$ is the amount of uncertainty the recipient has after communication, while $I(X, Y)$ is the amount of information the recipient receives during communication.

This understanding of information by Shannon implied what information can do, suggesting that information could be understood as negative entropy. However, this understanding of information cannot be valid beyond the field of communication theory as the calculation of entropy must strictly be limited within the domain of statistics. Furthermore, the concept of information in Shannon's theory considered merely the formative factor (signal waveform) of information, ignoring the meaning and value factors. The latter two factors, however, are absolutely needed for dealing with human activities and artificial intelligence systems.

In his integrative book "General System Theory", the integrator of systems theory Ludwig von Bertalanffy regarded entropy as a measure of disorder and hence information as a measure of order or of organization of a system [4]. In his book "Science and Information Theory", Leon Brillouin pointed out that information could be regarded as negative entropy [5]. These understandings concerning information are more or less the same as that defined by Shannon. This is because of the fact that the order of a system and the concept of negative entropy can very well be considered as different, and yet equivalent, expressions of probabilistic entropy under certain conditions.

Ross Ashby, the famous researcher in biological cybernetics/control, proposed his concept of information as the variety of a set [6] that is defined as the number of the elements in a set, or the logarithm of that number. This understanding seems somewhat too rough for fundamental studies of information.

In his monograph "Steps to the Ecology of Mind", an authority in psychology, Gregory Bateson, wrote that the technical term of information might be succinctly defined as any difference that makes difference in some later event [7]. He explained that the difference between some present state and some "preferred" state activates the corrective response. However, it also faces challenges, because 'No difference' itself is a kind of information. For example, if one asked a question "Is there any difference?" and answered "No difference", the statement "no difference" here evidently conveys 1 bit of information.

Of course, there have been many more 'definitions' of information that can be found in the literature, such as Burgin, summarized in [8]. Due to the limitations of the space of this paper, however, it is not possible, and also not necessary, to discuss each of them here. 
It is interesting to note that each of the definitions of information reviewed above has certain proponents and followers in the respective field. Nonetheless, no definition of information was unanimously accepted in all fields. If we review more definitions from different fields, it will show even larger discrepancy between these definitions.

Deep investigations reveal that the cause that led to the discrepancy in defining information can be attributed to the reductionist methodology, the main feature of which is "divide and conquer". This is the consequence of the fact that communication deals with the transmission of information, while cognition deals with information processing, and control deals with information utilization, etc. All of these fields are different, although they all belong to the information discipline. Following the reductionist methodology, the studies in the abovementioned fields have been isolated from each other in their research. As a result, different understandings on information were produced for different and isolated fields.

For finding a definition of information appropriate for all information fields, the "divide and conquer" methodology should be given up and a new methodology suitable for the information discipline, namely, the methodology of information ecology, should be introduced. For more details, see [9].

\section{System Methodology for Information and Ecological Model of Information Process}

According to the methodology of information ecology, the sections of perception, transmission, cognition, decision-making, and execution, all constitute an ecological chain/system, as in Figure 1.

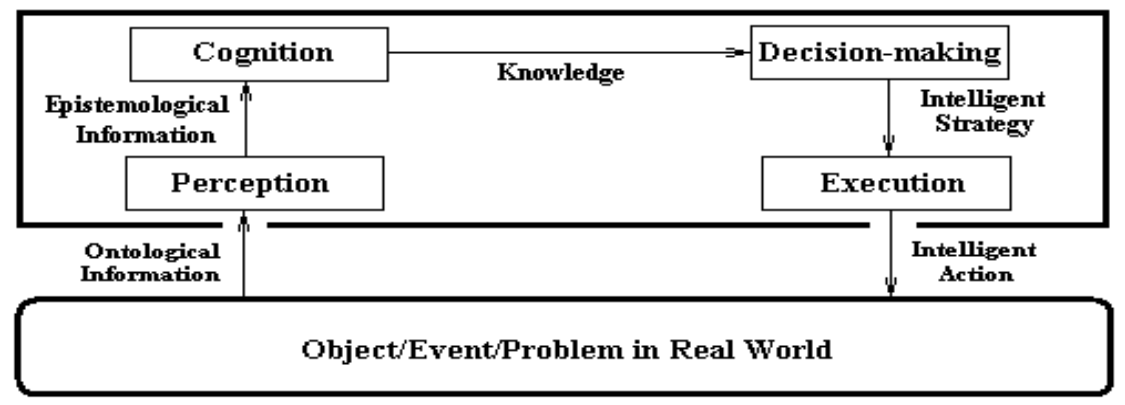

Figure 1. Model of Information Ecology [10].

This model stresses that for properly defining information, the notions of a system and ecology, rather than the notions of locality and isolation, should be utilized. Otherwise, the ecological chain of information process will be broken.

As one can clearly see from this model, the ontological information is generated and presented by the object, or problem, in the environment and will then be perceived through human perception, becoming epistemological information, the latter of which will in turn be conversed to knowledge through the process of human cognition and further be conversed to intelligent strategy and intelligent action through the process of decision-making and execution. Finally, the intelligent action will be reacted onto the object in the environment. The processes of ontological information, epistemological information, knowledge, intelligence strategy and intelligent action form an ecological chain, or equivalently ecological system, of information.

From the performed analysis, it follows that there are some important features of information.

Feature 1. Information is a popular phenomenon that exists in all possible areas in the real world and, hence, the same kind of phenomenon of information should universally be defined, and be unanimously applicable, for all fields of information studies.

Feature 2. Information is a kind of dynamics, and also a kind of productive phenomena, forming the ecological chain: information-knowledge-intelligence, under the framework of subject-object interaction. Therefore, the definition of information should observe the constraints of the ecological system. 
Comparing the aforementioned features inherent in information processes with the definitions of information that were reviewed in the previous section, it becomes obvious that the information definitions based on isolated sections of information process in the history are quite not reasonable and thus problematic.

Problem 1. Some existing definitions were given within one section, but were not suitable for others. The definitions of information set up by Shannon, Bertalanffy, Brillouin, and Ashby, respectively, fall into this category. They are all limited only to statistical events.

Problem 2. All existing definitions were given in isolated fields without considering the constraints imposed by ecological processes and interaction between subject sand objects in the environment.

As pointed out above, the real cause for the unsatisfactory definitions of information in history should be attributed to the use of the traditional methodology, "divide and conquer". This is due to the fact that, when dividing the ecological process of information into different components and different sub-processes, the information describing the interaction between sections or sub-processes will be completely lost and yet the interactive information process is the lifeline for information systems.

\section{New Definitions for Ontological and Epistemological Information}

Looking at the ecological model of information process shown in Figure 1, two categories of information exist. The first one, generated by an object/problem in the real world, is called ontological information and the second one, perceived from ontological information by a human subject, is called epistemological information. They are successively linked and closely interrelated to each other. Evidently, it is not sufficient to consider only one of them.

How do we precisely define the concepts of ontological and epistemological information then?

Considering the constraints, or requirements, from the ecological process of information, that is, the chain from information to knowledge and further to intelligent strategy and action as shown in Figure 1, the ontological information and epistemological information can be properly defined as follows.

Definition 1. Ontological information presented by an object is defined as "the state of the object and the pattern of the state transformations, which are presented by this object".

It can be seen from the Definition 1 and the model in Figure 1 that the ontological information generated and presented by an object depends only on the object itself in its environment and has nothing to do with the human subject. Ontological information is the original information, which transmits epistemological information through human perception.

Definition 2. Epistemological information perceived by subject from the ontological information is defined as the triad of the form (named syntactic information), the meaning (semantic information), and the utility (pragmatic information), while all these components are perceived by the subject from the related ontological information.

It is seen that epistemological information is the result of a subject's perception over the related ontological information. More explanations of the two successive definitions of information can be found in the literature [9].

\section{Concluding Remarks}

The major results presented in the article include the followings: 
(1) The mess in 'definitions' of information is due mainly to the employment of the reductionist methodology featured with "divide and conquer", which is powerful in the studies of physical science but not suitable for the studies of information science.

(2) For clearing up this mess, the system methodology for information, or the methodology of information ecology, has to be employed in the studies of information science.

(3) By employing the ecological model of information process in Figure 1, the definitions of ontological information and epistemological information with syntactic, pragmatic and semantic components are universally defined, such that they can serve as the unifying definitions of information.

(4) All the "definitions" mentioned above in section 2 can be regarded as special cases of the ones proposed in section 4 . The detailed explanations can be found in [9].

Funding: This research received no external funding.

Conflicts of Interest: The author declares no conflict of interest.

\section{References}

1. Wiener, N. Cybernetics; MIT Press: Boston, MA, USA, 1948.

2. Wiener, N. Cybernetics and Society; Houghton Mifflin Company: Boston, MA, USA, 1950.

3. Shannon, C. A Mathematical Theory of Communication. Bell Syst. Tech. J. 1948, 27, 379-423.

4. Bertalanffy, L. General System Theory; George Braziller: New York, NY, USA, 1973.

5. Brillouin, L. Science and Information; Academic Press Inc.: New York, NY, USA, 1956.

6. Ashby, R. An Introduction to Cybernetics; Wiley: New York, NY, USA, 1956.

7. Bateson, A. Steps to an Ecology of Mind; Jason Aronson Inc.: Northvale, NJ, USA, 1987.

8. Burgin, M. Theory of Information, Fundamentality: Diversity and Unification; World Scientific Press: Hackensack, NJ, USA, 2010.

9. Zhong, Y.X. Principles of Information Science; BUPT Press: Beijing, China, 1988.

10. Burgin, M.; Zhong, Y.X. The Methodology of information Ecology in the Context of Modern Sciences. Philos. Anal. 2019, 1, 119-136.

(C) 2020 by the authors. Licensee MDPI, Basel, Switzerland. This article is an open access article distributed under the terms and conditions of the Creative Commons Attribution (CC BY) license (http://creativecommons.org/licenses/by/4.0/). 